\title{
Vehicle License Plate Recognition Using MORPHOLOGY AND NEURAL NETWORK
}

\author{
Sneha G. Patel \\ Sardar Vallabhbhai Patel Institute of Technology, Vasad, 388 306, Gujarat, India \\ snehapatel11@gmail.com
}

\begin{abstract}
Automatic Identification of vehicles is a very challenging area, which is in contrast to the traditional practice of monitoring the vehicles manually. Automatic license plate (LP) recognition is one of the most promising aspects of applying computer vision techniques towards intelligent transportation system. In Location of the vehicle plate, a method of vehicle license plate character segmentation and extraction based on improved edge detection and Mathematical morphology was presented. In the first place, color images were changed into grey images, secondly through calculates the difference of each pixel and neighbourhood pixels to build up images edge and it can make the license plate stand out; Sobel operator is used to extract the edge of objects in image; then the algorithm applies the dilation and erosion mathematical morphology of binary images to get the image smooth contour. The segmentation result which is sent forward to LP recognition stage will improve further processing's efficiency. Neural Network is used to recognize the license plate character. Because of the accuracy of the plate region extraction, the character can be extracted exactly from the plate region.
\end{abstract}

\section{KEYWORDS}

License Plate Recognition, Back propagation, Neural Network, Median Filter.

\section{INTRODUCTION}

While the first industrial automatic system for Car License Plate Recognition (LPR) was introduced in the 80's, an outburst of commercial systems occurred in the 90s. Although that a lot of LPR systems are available in the market, the research and development still continues and new sophisticated solutions to plate localization, character segmentation and recognition appear.Vehicle's license plate recognition system has been a special area of interest in video surveillance area for more than a decade or so. With the advent of sophisticated video vehicle detection systems for traffic management applications, number plate recognition system finds wide varieties of places to fit itself beyond just controlling access to a toll collection point or parking lot [3]. It can now be integrated to the video vehicle detection systems which usually are installed in places of interest for intersection control, traffic monitoring etc., to identify vehicle that violates traffic laws or to find stolen vehicles. This paper presents a method of vehicle license plate Character recognition. The whole system into three following steps:

1- Plate location or finding location of plate in the vehicle image and cropping plate image from it.

2- Plate segmentation or cutting plate image to character's images.

3- Character recognition or convert character's images to final distinguished characters among them.

Due to the diversity of parameters involved in car images, License plate detection is considered the most crucial stage in the whole LPR system. In the past, a number of techniques have been proposed for locating the desired plate through visual image processing. Now there are some algorithms about the locating of license plate, such us the methods based on color feature [4],

DOI : 10.5121/ijci.2013.2101 
edge extracting [6-8], histogram analysis [9], symmetry [11], morphological operators [12],.In a method of Chinese license plate recognition has been proposed that structural verification is used for plate locating and projection segmentation is exploited for incising plate image into character's images. Sobel operator is the first and best-known choice for most LPD algorithms to convert the grayscale scene image to the gradient image at the initial stage.[12] In general, conventional LPD algorithms focus only on vertical edge detection through vertical Sobel operator, because the horizontal edge density of license plates is not more outstanding than that of the surrounding background objects. Character recognition of the number-plate is a fairly well developed field in computer vision in which template matching and neural networks are often used and can produce satisfactory results [14-15]. However, template matching has its drawbacks in some aspects comparing with neural networks. For example, when characters of number-plate are segmented, neural network approach is preferred to template match one. The paper is organized as following way. Section I is of introduction of whole system. We shall introduce the proposed recognition algorithm in section II to V. Experiment and Conclusion will be shown in Section VI and Section VII respectively.

\section{PRE-PROCESSING}

Preprocessing mostly is necessary to facilitate further high performance recognition, in this proposed methodology, the character is binarized and the noise is eliminated in the preprocessing stage.

\subsection{Grey Conversion}

We have taken color image pf car clearly showing its License plate for experiment.We firstly convert this RGB color input image to a 256 grayscale image using formula (1).

$G R E Y=J(:,:, 1)+J(:,:, 2)+J(:,:, 3) \ldots \ldots \ldots \ldots(1)$

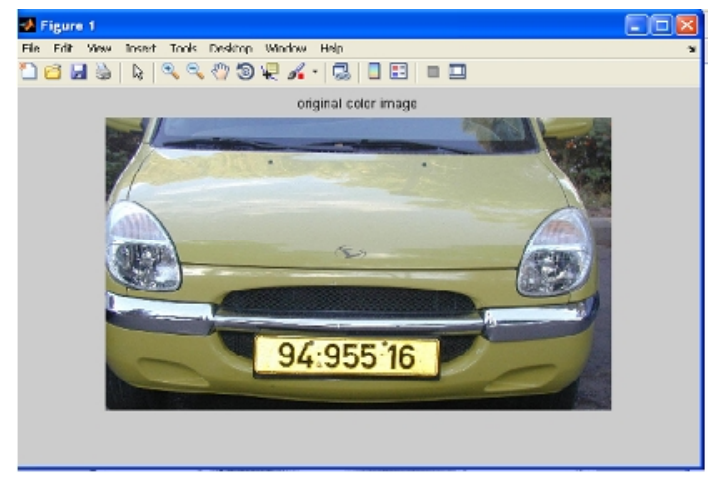

Figure 1. Original color image

The grey-scale could take every pixel of the picture to a number between 0-255 and the purpose of the binarization is to take every pixel into the number 0 or 255 . To remove the tonal variation between Red, Green and Blue channels of input images and converting it into grey scale flatness to a single hue. 


\subsection{Median Filtering}

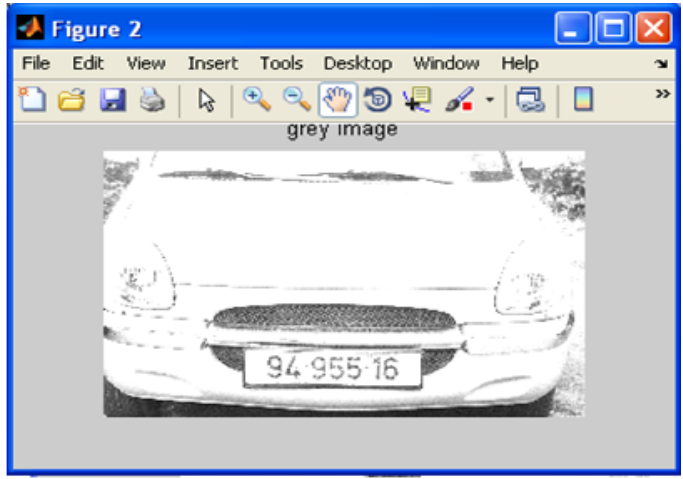

Figure 2. Grey image

It is inevitable for containing noises of original image. We use median filtering to eliminate the noises. Using median filtering not only can eliminate the noises, but also make the high frequency more concentrated. There by, it is beneficial for us to detect the edges in images. The salt and pepper characteristic of vehicle license plate is presented, So Median filter is best known choice for removing such noise.

\section{License Plate Extraction}

To extract the license plate, we mainly utilize the characteristic of the plate in vehicle images like the color in the region is blue. We project the binary difference image horizontally and vertically, properly smooth these two projection curves, and search their peaks to find the accurate rectangle that includes the plate.[7]

\subsection{Opening and Closing}

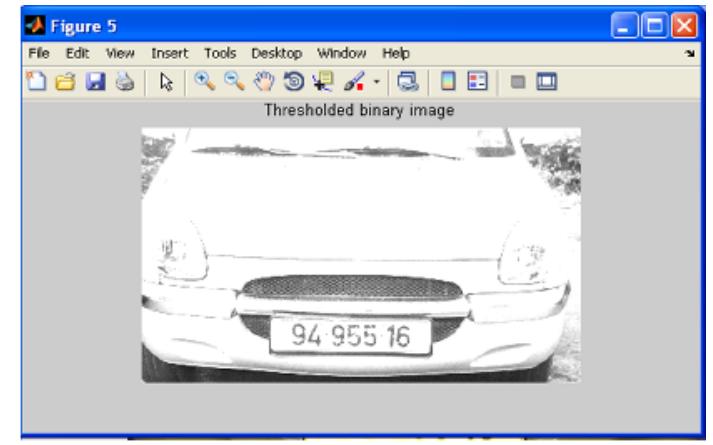

Figure.3. Thresholded Binary image

Opening generally smoothes the contour of an object, breaks narrow isthmuses and eliminates thin protrusions.Closing also tends to smooth sections of contours but, as opposed to opening, it generally fuses narrow breaks and long thin gulfs, eliminates small holes, and fills gaps in the contour [10].

The opening of set $A$ by structuring element $B$, denoted $A_{-} B$, is defined as $A \_B=\left(A \_B\right) \oplus B$

Thus, the opening of $A$ by $B$ is the erosion of $A$ by $B$, followed by a dilation of the result by $B$.

Similarly, closing is defined as

$A \cdot B=(A \oplus B) \_B$ 


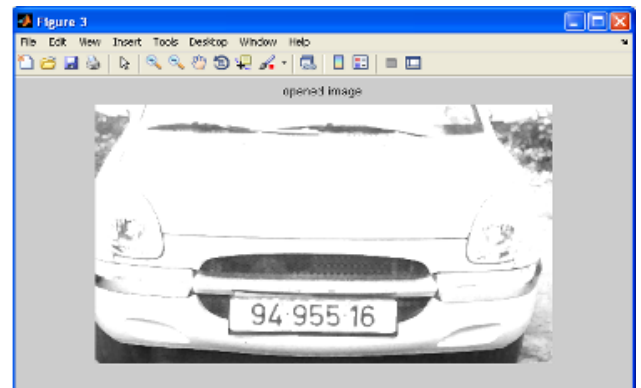

Figure 4. Opened image

This says that the closing of $A$ by $B$ is simply the dilation of $A$ by $B$ followed by the erosion of the result by $B$.

\section{Character Segmentation}

Character segmentation is an important stage in many license plate recognition systems. There are many factors that cause the character segmentation task difficult, such as image noise, plate frame, rivet, and rotation and illumination variance [13]. Object segmentation is an essential task in computer vision and object recognitions. Image segmentation is the process of partitioning a digital image into multiple regions or sets of pixels [6]. These partitions represent different objects in the image, usually having the same texture or color. Segmentation is quite essential to image feature extraction and subsequent classification of the resultant features.This step is very significant due to overlapping characters that form the license plate. There are three main forms of characters that are overlapping vertically, ligature, diacritics, horizontal overlap, and two connected characters. The task will be more difficult for those different forms of Which are joined.

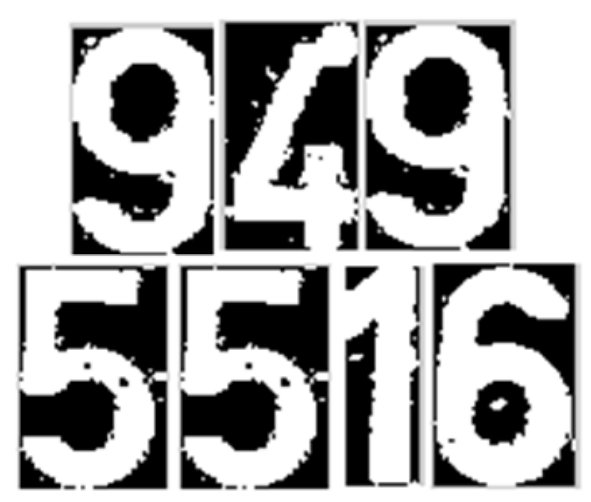

Figure 5. Segmented characters of License plate.

\section{Character Recognition}

The two methods are template-matching method and neural network method. Here we are evaluating neural network method. Character recognition final step in vehicle license plate detection and recognition is reading of single characters and numbers. This step is very important for example at the entrance to car-park or for the police for stolen cars search. Single elements on license plate must be segmented and analyzed. The analysis is called as Optical Character Recognition (OCR) using ANN [13]. 
International Journal on Cybernetics \& Informatics ( IJCI) Vol.2, No.1, February 2013

\subsection{Artificial Neural Networks}

Artificial neural networks are statistical models of real world systems which are built by tuning a set of parameters. These parameters, known as weights, describe a model which forms a mapping from a set of given values known as inputs to an associated set of values, the outputs. The process of tuning the weights to the correct values -training- is vehicleried out by passing a set of examples of input-output pairs through the model and adjusting the weights in order to minimize the error between the answer the network gives and the desired output. Once the weights have been set, the model is able to produce answers for input values which were not included in the training data. The models do not refer to the training data after they have been trained; in this sense they are a functional summary of the training data $[1,10]$.

The use of artificial neural network (ANN) in OCR applications can dramatically simplify the code and improve quality of recognition while achieving good performance. Another benefit of using neural network in OCR is extensibility of the system i.e. ability to recognize more character sets than initially defined. In the character recognition algorithm using neural networks, the weights of the neural network were adjusted by training it using back propagation algorithm. The size of each character is 28-by-18 pixels which are arranged column wise to give 504 _ 1 arrays as input. In order to train the neural network, we have created different sets each containing digits from 0 to 9 . This is called Block training. The complete net work was implemented as a library, which was statically tied to the project. This helped to isolate the neural network code from the rest of the preprocessing and segmentation code [14]. It also helped to reduce the memory required for the program.Character recognition of the number-plate is a fairly well developed field in computer vision in which template matching and neural networks are often used and can produce satisfactory results However, template matching has its drawbacks in some aspects comparing with neural networks. For example, when characters of number-plate are segmented, neural network approach is preferred to template match one due to more computation cost of the later method [6].

As a very powerful technique for pattern recognition problems, it is not surprising that neural networks became the basis of a number of such systems [15].

\subsection{The Back Propagation (Bp) Algorithm}

The back propagation is a widely used algorithm, and it can map non-linear processes. It is a feed forward network with the one or more hidden layers. The elementary architecture of the back propagation network has three layers. There are no constraints about the number of hidden layers. Back propagation is a systematic method for training multilayer artificial neural net works. It has a mathematical foundation that is strong if not highly practical.

Using neural network has advantage from existing correlation and statistics template techniques [5] that allow being stable to noises and some position modifications of characters on license plate. The network must memorize all the Training Data (36 characters). For the validation of the network we have built a program that reads the sequence of characters, to cut each character and resize it and put the result [16].

\subsection{Training}

Once the network has been initialized and the training input space prepared the network is ready to be trained. 


\section{EXPERIMENTAL RESULTS}

Proposed method was implemented using MATLAB version 7.11 and configuration of personal computer includes: windows XP, Intel(R) Pentium 4, $1.80 \mathrm{GHz}$ processor with 1GB RAM. Images of car are taken from internet database. Experiments are done on cars of various colours.

The below figures are segmented characters of License plate of car image.
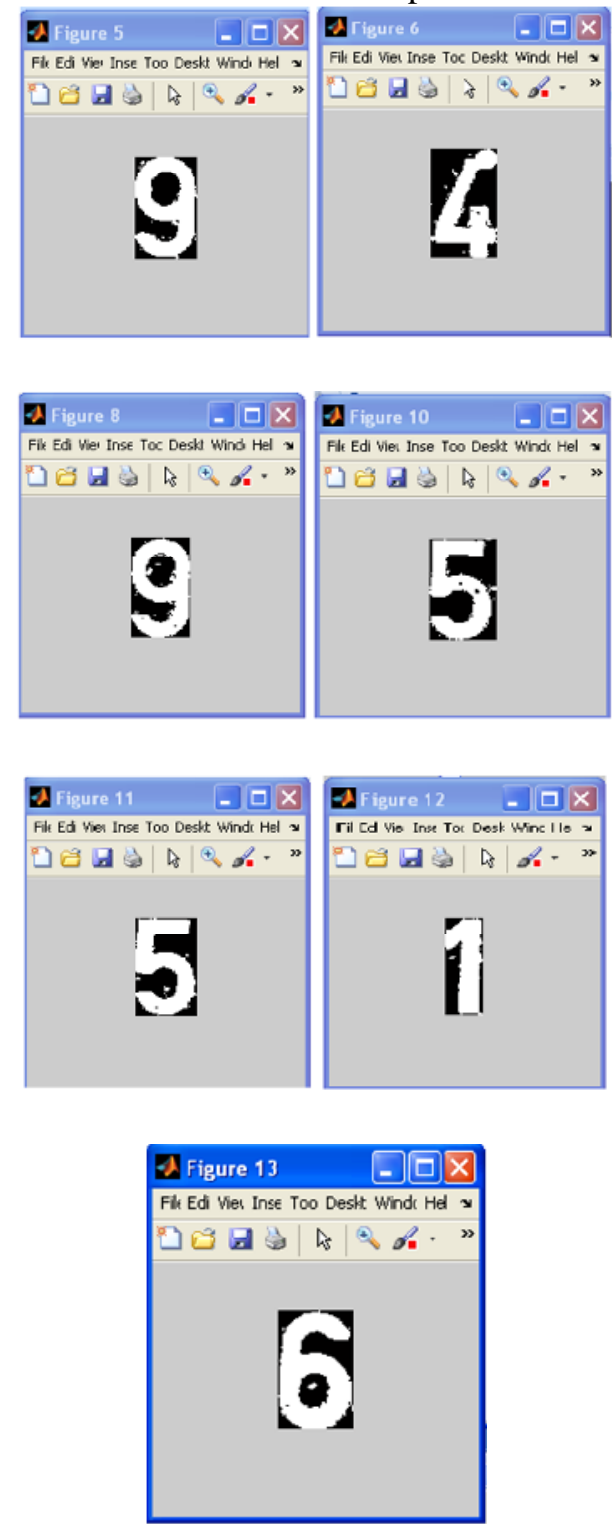

\section{Conclusions}

This paper introduces a neural network approach for automatic identification of vehicle License plate. Recognition of character are better then Template matching method. If the accuracy of the results is a critical factor for a vehicle number plate recognition application, then the network having many hidden layers should be used but if training time is a critical factor then the network 
having single hidden layer should be used.The proposed approach of license plate recognition can be implemented by police to detect speed violators, parking areas, highways, bridges or tunnels. Also the prototype of the system is going to be integrated and tested as part of the sensor network. Currently there are certain restrictions on parameters like speed of the vehicle, script on the number plate, cleanliness of number plate, quality of captured image, skew in the image which can be removed by enhancing the algorithms further.

\section{REFERENCES}

[1] Ahmad and Mohammad,2009 ," Efficient Farsi License Plate Recognition”, IEEE.

[2] Q. GAO,et al.,Aug. 2007,” License Plate Recognition Based On Prior Knowledge “, Proc. IEEE Inter. Conf. Automation and Logistics Jinan, China, pp.2964-2968.

[3] Ahmad Radmanesh, June 2005, “A Real Time Vehicle's License Plate Recognition System" , Proceedings of the IEEE Conference on Advanced Video and Signal Based Surveillance (AVSS'03) Vol 4, pp.159-167.

[4] Zhu Wei-gang,Aug. 2002, "A study of locating vehicle license plate based on color feature and mathematical morphology, Signal Processing “, 6th International Conference on, pp.748-751, Vol.1.

[5] Bernard and Galit, Aug. 2002, "Wavelet-Based Monitoring for Disease Outbreaks and Bioterrorism: Methods and Challenges, Signal Processing”, 6th International Conference on, Vol.1.

[6] Rafael and Richard,2009, "Digital Image Processing", $3^{\text {rd }}$ ed. Prentice-Hall Inc.

[7] Hui Wu and Bing Li,2011, "License Plate Recognition system”, IEEE.

[8] K. Kanayama,et al.,1991, "Development of vehicle-license number recognition system using realtime image processing and its application to travel-time measurement", Processing's of IEEE Vehicular Technology Conference, pp.789-804.

[9] D.U. Cho and Y.H. Cho, "Implementation of pre-processing independent of environment and recognition of car number plate using histogram and template matching", The Journal of the Korean Institute of Communication Sciences, 23(1)

[10] Shaohong Wu,October 2011, "A Novel Accurately Automatic License Plate Localization Method" ICEES, pp.155-160.

[11] D. S. Kim, and S. I. Chien, 2001, "Automatic car license plate extraction using modified generalized symmetry transform and image warping”, Proc. IEEE Int. Symp. On Industrial Electronics, Vol. 3, pp.2022-2027.

[12] Ming-Kan Wu, et al., 2009, “2-Level-Wavelet-Based License Plate Edge Detection", 5 ${ }^{\text {th }}$ International Conference on Information Assurance and Security, ICIAS, pp.385-388.

[13] Seyed Hamidreza, et al., 2011, "Extraction and Recognition of The Vehicle License Plate for Passing under outside Environment”, IEEE, European Intelligence and Security Informatics Conference, pp.234-237.

[14] Parul Shah, et al.,2009, “OCR-based Chassis-Number Recognition using Artificial Neural Networks", ICVES 2009.

[15] Feng Yang, and Fan Yang, "Character Recognition using Parallel BP Neural Network", IEEE.

[16] A. Akoum, et al.,2009, Two Neural Networks for License Number Plates Recognition”, Journal of Theoretical and Applied Information Technology.

[17] Subhash Tatale, and Akhil Khare,Sept 2011, "real time anpr for vehicle identification using neural network"k, International Journal of Advances in Engineering \& Technology, IJAET ISSN: 22311963- 262, Vol. 1, Issue 4, pp.262-268.

\section{Authors}

Sneha G. Patel, M.E. [E \& C Engineering] Student, Department of Electronics \& communication, Sardar Vallabhbhai Patel Institute of Technology, Vasad, 388 306, Gujarat, India. 